\title{
C-RDB: Response
}

\subsection{INTRODUCTION}

We'd like to thank the discussants for their thoughtful contributions. Their remarks cover many of the points we have previously encountered and raise interesting new ideas. They also offer a glimpse into the diversity of views and innovations encouraged by the evergrowing world of statistics education.

Cobb, in particular, shared many kind words. We are thankful that he pointed out the agreement between OpenIntro Statistics and the AP and GAISE guidelines, which we think contributes to the broad applicability of the book. We especially appreciate his suggestion that making OpenIntro Statistics freely available and customizable has the potential to revolutionize education. We are both flattered and hopeful!

It is a special treat to participate in such a rich discussion. Before continuing, we want to emphasize a few key points. First, we agree with the discussants that student learning is the top priority. Second, we think both the quality and accessibility of resources are factors in learning. Lastly, we believe the future will see a variety of approaches to textbooks all flourishing in parallel.

\subsection{HIGH QUALITY, LOW COST}

Velleman commented that "one consistent argument for e-texts across these and all other articles on the subject is that they cost so much less than paper and print texts that this alone makes them a good choice and our inevitable future". He later questioned the validity of such a position; so do we. We do not believe that a textbook having the virtue of being free is necessary or sufficient for it to be a good choice. However, we do believe free is a feature that enhances student learning by expanding accessibility.

We appreciate Velleman's skepticism about resources being simultaneously high quality and free, and this is a genuine concern. However, there are many examples of free resources that are of exceptional quality: Wikipedia and StackExchange (e.g. stackoverflow, CrossValidated) quickly to mind. Cornerstones of such successful open-source projects, on which OpenIntro has been modeled, include many thousands of volunteer hours, extensive and personal communication with end users (instructors and students), and diversity of products (including a paperback version available on Amazon at cost). 
One advantage of a free textbook is that it immediately facilitates a comparison. We are extremely enthusiastic about instructors reviewing textbooks, choosing a couple, and then performing an empirical comparison. For example, a teacher may cite readings or assign homework from both a free textbook and a currently used textbook without adding an extra financial burden to students. Interesting questions to explore might include the clarity of explanations in each textbook, whether the text was intellectually engaging, whether the resource was accessible, and many other characteristics. Ideally, every textbook adoption would employ such a rigorous comparison.

Velleman also noted, "Textbooks, like pharmaceuticals, are selected by experts who are not the ones who pay for them or use them." We agree. That is why we are not proposing that students choose a textbook. Rather, we are suggesting instructors give students the opportunity to compare textbooks and provide feedback. After all, if the goal is student learning, we should be assessing the quality of a textbook by how well it teaches, just like we assess the effectiveness of a pharmaceutical by whether or not the patients who use them get better.

\subsection{Recognize diversity}

Diversity is another important consideration. Velleman referenced statistics showing that a majority of professors prefer print texts. We recognize the demand for print copies, which is why we have always made a print edition of OpenIntro Statistics available. However, we also understand that some instructors and students appreciate easy and unrestricted access to electronic copies.

Students also differ in their financial backgrounds. A recent study in Florida found that a majority of students have skipped the purchase of a required textbook because of its high cost (Florida Virtual Campus 2012). We worry that the failure to recognize and respond to the diverse financial situations of students will ultimately disqualify many students from learning as much as they would like. This is why we strongly believe cost should be an important consideration when selecting a textbook. Velleman commented that cutting costs on textbooks would be "a relatively small amount of savings in an expensive college education". However, this is not completely accurate. For example, in the community college setting, switching from a traditional textbook (for example, one at \$150) to a free alternative, would in many cases reduce the direct cost of that course by $25 \%$ or more.

\subsection{Production support}

Velleman noted the many challenges associated with publishing and promoting a textbook, 
and suggested that e-texts require similar support. We agree partially, but not completely. OpenIntro is fortunate - a growing number of talented volunteers have been eager to support this project. For example, Velleman mentioned the importance of design. We take design very seriously, and we benefit tremendously from the careful hands of our experienced design specialist Meenal Patel, a graphic designer behind the textbook as well as www.openintro.org. Indeed, this is just one example. Other contributions, including support in pedagogical considerations, content generation, fund raising, legal advice, and business strategy are provided by equally enthusiastic and talented people and organizations

- many of whom are acknowledged in the About section of our homepage. To reduce the burden on our volunteer network, we also try to identify areas where support is not critical. For example, the public domain is a rich arena from which many of our resources have been drawn, including, notably, photographs and data sets. Lastly, some features of the traditional publishing house, most notably paying a large staff of sales representatives, are not included in the OpenIntro model. Collectively, these provide the basis for savings that make the OpenIntro project feasible.

Utts asked what has allowed us time to create these materials. She mentioned, as we have also found, that universities do not generally provide time for such projects unless they are grant funded, and she noted textbooks are traditionally written over nights and weekends. That's exactly how OpenIntro Statistics was written as well!

\subsection{CONCLUSION}

Rossman and Chance wrote that they "doubt the role of traditional publishers will be diminished much [in the coming decades]." We agree. The traditional publisher provides a collection of services (and avenues for financial rewards) that are very useful to helping and incentivizing the writing of high quality textbooks. We believe the two models for introductory textbooks - traditional publishers and open-source publishers - will exist in parallel in the coming decades. Indeed, one might draw an analogy to water: you can pay for bottled water, or you can get it freely from the tap. Both will serve the same function. However, depending on your resources, taste, and other factors, you might prefer one over the other.

The fundamental difference between the adoption of free and non-free textbooks is that free resources can be tested (and student feedback gathered) before adoption, which is often unrealistic with expensive textbooks. Like many people have done by comparing bottled water to tap water, we ask instructors to compare for themselves, and decide which they and their students prefer. 


\subsection{REFERENCES}

Florida Virtual Campus (2012). "2012 Florida Student Textbook Survey Executive Summary." 